\title{
La Cultura ambiental desde la participación ciudadana para el desarrollo de la conciencia crítica en la ciudadanía Lojana
}

\section{The Environmental Culture from the citizen participation for the development of the critical conscience in Loja's citizens}

Aurita Geovania Gonzaga Figueroa

Universidad Nacional de Loja, Ecuador

Autor para correspondencia: aurita.gonzaga@unl.edu.ec

Fecha de recepción: 02 de octubre de 2018 - Fecha de aceptación: 15 de octubre de 2018

Resumen: El presente artículo propone una alternativa sobre cómo mejorar la cultura ambiental en las personas para así poder obtener y convivir en un ambiente sano. Un elemento esencial es lograr en los actores sociales el desarrollo de su conciencia crítica para que los impactos delhombre sobre elmedioambiente disminuyan, para garantizar el perfeccionamiento de una cultura ambiental que le proporcione los conocimientos, habilidades y valores necesarios para su transformación personal y social. El objetivo general de la investigación fue lograr la formación de una cultura ambiental en los comerciantes del mercado mayorista "Gran Colombia" Loja-Ecuador, a través del manejo integral de los residuos sólidos generados en la zona. La propuesta fue concebida desde la metodología de investigación-acción-participación, que se constituye en una vía efectiva que garantiza la potenciación de procesos de desarrollo de conciencia crítica, participación activa de los implicados en la determinación y solución del problema y la elaboración de proyectos sociales que conducen desde la formación de una cultura ambiental a mitigar los impactos ambientales desde el protagonismo de la ciudadanía. La evaluación de los resultados del proyecto de intervención para contribuir a la formación de la cultura ambiental de los comerciantes, permitió el desarrollo de la toma de conciencia crítica de los comerciantes sobre los problemas de su entorno, específicamente la problemática del mal manejo de residuos sólidos. Desde esta perspectiva de investigación-acción-participación se evidenciaron cambios favorables en cuanto a la conciencia crítica, y se aprecia la disposición a la participación en las tareas para solucionar los problemas actuales e impedir la contaminación de los sectores aledaños al mercado y la cultura ambiental de los comerciantes para solucionar este deterioro. A través de este programa se establecen proyectos dirigidos a: reciclaje, aplicación de bonos, educación ambiental basada en el manejo y aprovechamiento de los residuos sólidos, almacenamiento selectivo, instalación de un centro de acopio y por último el de fomentar la formación de una organización comunitaria que ejerzan el aprovechamiento de residuos sólidos.

Palabras claves: cultura ambiental; participación ciudadana; manejo integral de residuos sólidos Abstract: This article proposes an alternative on how to improve the environmental culture in people in order to obtain and live in a healthy environment. An essential element is to achieve in the social actors the development of their critical conscience so that the impacts of man on the environment diminish, to ensure the improvement of an environmental culture that provides the knowledge, skills and values necessary for personal and social transformation. The general objective of the research was to achieve the formation of an environmental culture in the "Gran 
Colombia" wholesale market merchants in Loja-Ecuador, through the integral management of solid waste generated in the area. The proposal was conceived from the methodology of researchaction-participation, which is an effective way to guarantee the empowerment of processes of critical awareness development, active participation of those involved in the determination and solution of the problem and the elaboration of social projects initiatives that lead from the formation of an environmental culture to mitigate the environmental impacts from the leadership of citizenship. The evaluation of the results of the intervention project to contribute to the formation of the environmental culture of the merchants, allowed the development of critical awareness of merchants about the problems of their environment, specifically the problem of poor solid waste management. From this perspective of research-action-participation, favorable changes were evidenced in terms of critical awareness, and the willingness to participate in tasks to solve current problems and prevent contamination of the sectors surrounding the market and environmental culture of the merchants to solve this deterioration is appreciated. Through this program, projects are established aimed at: recycling, bond application, environmental education based on the management and use of solid waste, selective storage, installation of a collection center and finally the promotion of the formation of an organization that exercise the use of solid waste

Key Words: environmental culture; citizen participation; integral management of solid waste

\section{Introducción}

Novo (2009) señala que en el contexto mundial, el desarrollo de una cultura ambiental supone un cambio de concepción del individuo sobre sí mismo y de su lugar en el mundo, y consecuentemente con respecto a los demás, con la sociedad y con la naturaleza y que debe apropiarse del conocimiento de una realidad compleja, aprender a interactuar con ella de otro modo, pero sobre todo debe reorientar sus fines, sin abandonarlos, considerar al hombre como individuo, especiey miembro de grupos sociales, lo incorpora al mundo desde ámbitos múltiples. Guadiano (2000) indica que las relaciones hombre-sociedad-naturaleza son condicionadas por la cultura ambiental, determinan la orientación de la dimensión ambiental de la actividad humana expresada en las políticas de los Estados, de las instituciones productivas, de servicio, de investigación, sociales, educativas, artísticas, culturales, y en el comportamiento social. Duque (2011) menciona que el concepto de educación ambiental no se ha mantenido estático, el mismo se ha modificado, precisamente en correspondencia con la evolución de la idea de medio ambiente. En sus inicios, la atención se centró en posiciones naturalistas por creer que el medio ambiente era solo lo natural: la conservación de los recursos naturales, la protección de la flora y la fauna, de los suelos y las agua Paulatinamente, el propio desarrollo ha llevado a abandonar las tendencias ecologistas e incorporar al medio ambiente las dimensiones tecnológicas, políticas, económicas y socioculturales, las cuales son fundamentales para entender las relaciones de la humanidad con su naturaleza y así poder gestionar, proteger y conservar el medio ambiente. Mora (2013) \& Frabboni et al (2006) sostienen que una de las alternativas más importantes para mitigar los impactos de los problemas ambientales está en el proceso de educación de la ciudadanía. Para comprender este proceso es necesario explicar la concepción de educación asumida para la formación de la cultura ambiental en la población. La educación es un proceso de socialización, inherente a la existencia de la sociedad y puede ser orientado al desarrollo pleno de las cualidades de la personalidad del sujeto individual y 
social como premisa para la apropiación de los conocimientos, las capacidades, los sentimientos, las emociones, las convicciones, la voluntad y los valores en general, como afirma (Molina, 2003).

En el proceso de formación de la cultura ambiental, no es posible desestimar todos los demás factores de influencia de la situación social en la cual se desarrolla el individuo; por eso, en este proceso también influyen la familia, la comunidad, los medios de comunicación masiva, en fin, la sociedad en general; esta idea está conceptualizada en la definición de educación en su sentido amplio (Maass, 2005). Escudero \& Martínez (2011) mencionan que hay especial interés a la educación ambiental como una forma específica de educación la cual permite desarrollar una conciencia crítica sobre la problemática ambiental, en este sentido, se comprende como crítica, la capacidad de romper un paradigma de mero espectador de los hechos a una actitud militante, activa, capaz de captar la génesis y la evolución de los problemas ambientales.

Arboleda (2014) ratifica que la cultura ambiental es el reconocimiento del paso del ser humano por la vida y su ambiente, por lo tanto está en constante cambio y que para desarrollar propuestas educativas, han de tenerse en cuenta los fundamentos epistemológicos y sociopolíticos del modo de pensar la relación sociedad naturaleza por el individuo de hoy. Guerrero et al (2010) señalan que el objetivo de la educación ambiental es lograr la formación de una cultura ambiental y la participación ciudadana en la preservación del medio ambiente, para garantizar la preparación adecuada de la formación de las comunidades locales, en la gestión ambiental de esos territorios y no detener su crecimiento «desarrollo», para dejar de creer que solo se puede desarrollar destruyendo parte de lo existente en el ámbito local y pueden condicionar la real participación de la población. Ello se manifiesta en el enquistamiento del poder, la anulación del protagonismo y la autonomía ciudadana. A nivel de la vida cotidiana, no pocas veces sucede que la mayoría de las personas tiendan a delegar en otros, tareas que ellos deben y pueden realizar.

\section{Metodología}

\section{Área de Estudio}

La investigación se desarrolló en el Mercado Mayorista “Gran Colombia” que se encuentra ubicado en la Av. Gran Colombia y Tulcán al norte de la cuidad de Loja, en el cantón y provincia de Loja. El Área está situada a una altitud de $2100 \mathrm{msnm}$., posee un clima temperado-ecuatorial subhúmedo con lluvia de $900 \mathrm{~mm}$ anuales y una temperatura media del aire de $16{ }^{\circ} \mathrm{C}$. El período con menor temperatura media se extiende de junio a septiembre, y julio es el mes más frío $14^{\circ} \mathrm{C}$. (PNUMA, et al, 2007). 


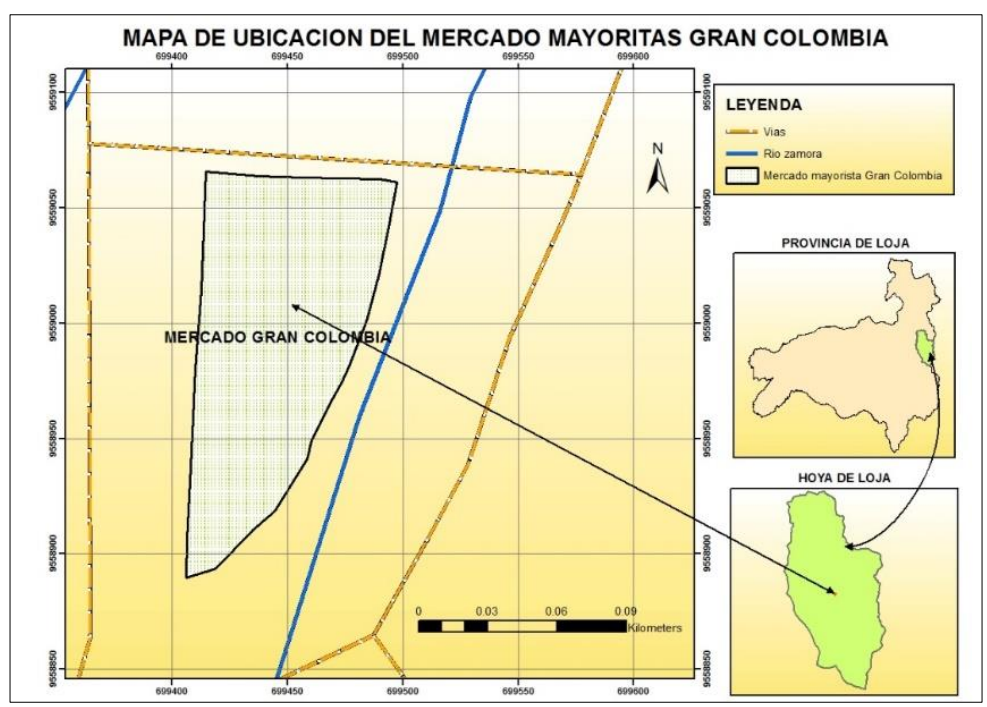

Figura 1. Mapa de ubicación de mercado Gran Colombia.

La investigación se llevó a cabo desde el punto de vista metodológico cuantitativo y cualitativo dado que emplea métodos que tributan a ambas perspectivas metodológicas a través de procesos inductivos y deductivos, con el uso de las herramientas participativas de investigación - acción - participación (IAP) según Balcazar (2003). Se encuestaron a 30 comerciantes del mercado definidas por secciones que corresponde a la venta de: frutas y verduras, tienda de abarrotes, cárnicos, venta de flores, prendas de vestir y comida; con un total de cinco encuestas por sección, seleccionadas aleatoriamente para una buena distribución de los datos arrojados por los encuestados, y tener así una alta confiabilidad de la información que serán importantes para la toma de decisiones y establecer propuestas para mejorar el manejo de los residuos en el Mercado Mayorista "Gran Colombia". Una vez aplicadas las encuetas se tabularon cada una de las preguntas y se hizo la respectiva representación de la información.

\section{Resultados}

Una vez aplicada la encuesta se obtuvo los siguientes resultados

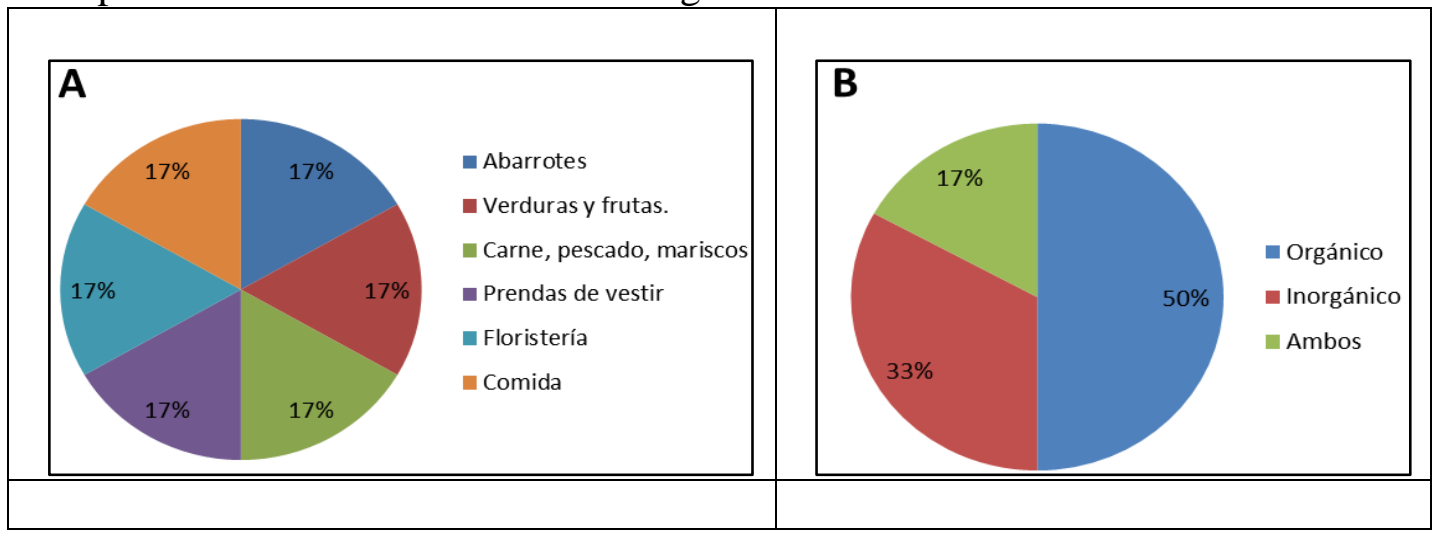




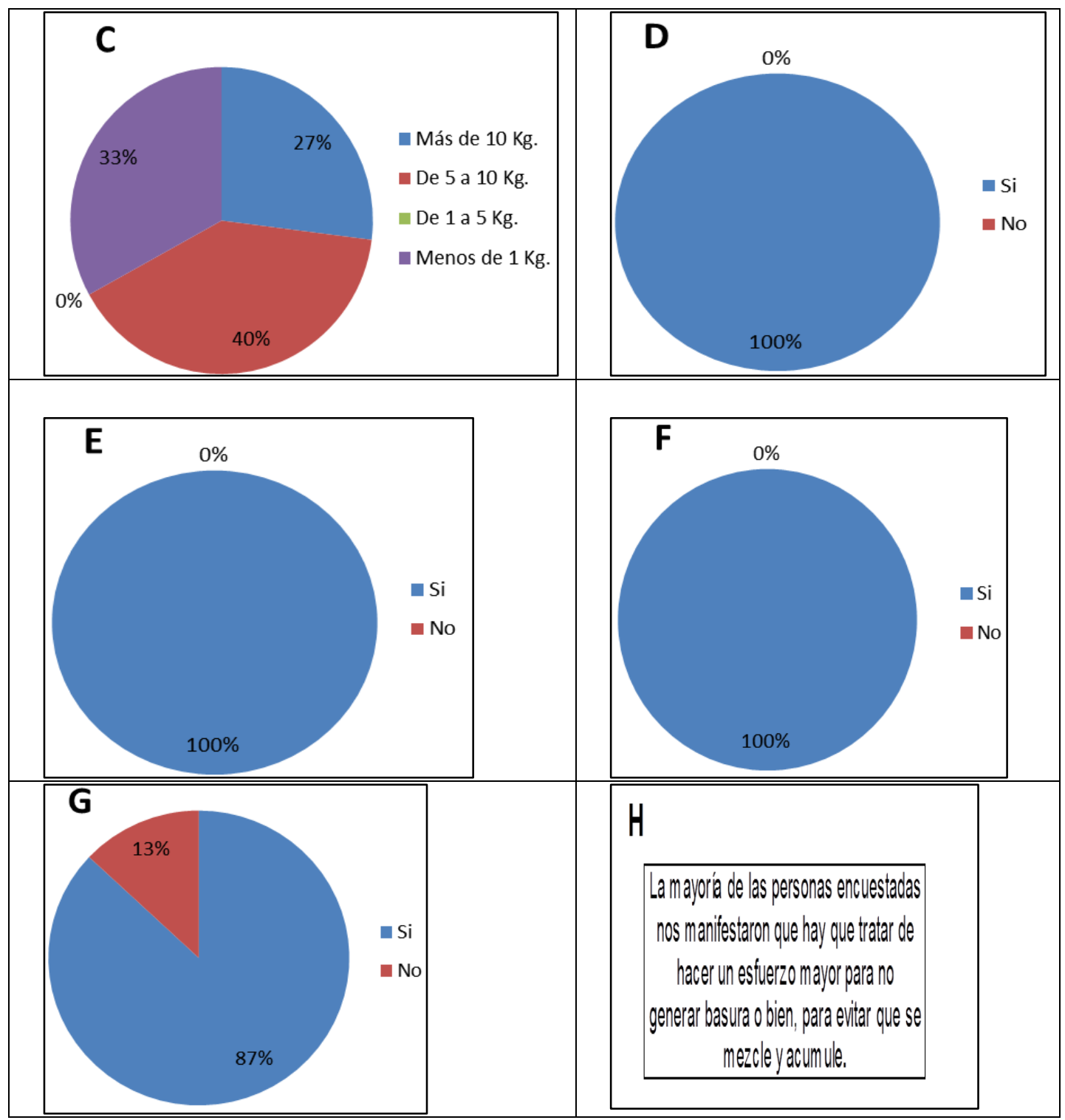

FIGURA 1. Representación gráfica de las preguntas aplicas en la encueta. A) ¿Qué tipo de productos usted comercializa?, B) ¿Qué tipo de residuos produce su negocio?, C) ¿Qué cantidad de residuos produce diariamente aproximadamente?, D) ¿Cree que existe un buen manejo de los residuos en el Mercado Mayorista?, E) ¿Existe algún tipo de control dentro del mercado Mayorista?, F) ¿Tiene algún conocimiento de lo que es el reciclaje?, G) ¿estaría dispuesto a recibir charlas, talleres sobre el buen manejo de los Residuos Sólidos?, H) estaría dispuesto a recibir charlas, talleres sobre el buen manejo de los Residuos Sólidos?.

En resumen los encuestados manifestaron que con respecto al tipo de residuos sólidos generados mencionaron que generan residuos orgánicos ya que su trabajo es de expender frutas y verdura por ende generando gran cantidad de este tipo de basura, lo mismo ocurre con los que venden productos cárnicos y los de la venta de floristería, así mismo señalaron que existe un buen manejo de los residuos sólidos tanto en el sector interno del mercado, así como también al momento de depositarlos en los contenedores respectivos y que existe un buen control por parte 
de las autoridades y personal encargado con respecto al manejo de desechos; y finalmente la mayoría de los encuestados si tienen conocimiento alguno de lo que es el reciclaje y cuán importante es por ejemplo ellos nos supieron contestar que el reciclaje se trata de separar los componentes de la basura en orgánicos e inorgánicos, además de clasificar los componentes inorgánicos en papel, cartón, vidrio y metales y procesar cada material de desecho con un tratamiento adecuado.

\section{Discusión}

Para Castillo (2010) el sistema de desarrollo dominante, con sus políticas neoliberales y enfoques mercadocéntricos contribuye a agravar los problemas socio-ambientales, bajo un contexto de la globalización del mercado. Por eso, la preocupación por el manejo sustentable del ambiente bajo el enfoque de cultura ambiental, hace imperiosa la necesidad de estructurar una educación ambiental que forme e informe acerca de esta problemática. En este sentido, la educación ambiental viene a constituir el proceso educativo que se ocupa de la relación del ser humano con su ambiente (natural y artificial) y consigo mismo. La educación ambiental puede y debe ser un factor estratégico que incida en el modelo de desarrollo establecido para reorientarlo hacia la sustentabilidad y la equidad. "Para contribuir con eficacia a mejorar el ambiente, la acción de la educación debe vincularse con la legislación, las políticas, las medidas de control y las decisiones que los gobiernos adopten, en relación con el ambiente humano" (UNESCO, 2004). La educación ambiental atañe a toda la sociedad, debe tener un enfoque amplio, para potenciar un pensamiento crítico e innovador, que sea capaz de formar una opinión acerca de los problemas socio-ambientales. Con la educación ambiental se pretende fomentar el compromiso de contribuir al cambio social, cultural y económico, a partir del desarrollo de valores, actitudes y habilidades que permitan a toda persona formarse criterios propios, asumir su responsabilidad y desempeñar un papel constructivo (González \& Gaudiano, 2003). Según Zubiet \& Delfino (2010) manifiestan que un programa de educación ambiental es la noción que permite nombrar a la serie de acciones organizadas con el objetivo de mejorar las condiciones de vida en una región. Además, es una referencia para la implementación de los servicios interpretativos, su distribución en un territorio considerado, y la elección de los mensajes más relevantes a transmitir a la comunidad y que por mínimo debe contar con las siguientes etapas:

Pintado (2011) menciona que el incorrecto manejo de los residuos sólidos afecta significativamente el bienestar y la salud de la población. Los riesgos de contraer enfermedades o de producir impactos ambientales adversos varían considerablemente en cada una de las etapas por las que atraviesan el manejo los residuos sólidos. Según Meira (2013) la generación y almacenamiento de residuos sólidos en el hogar puede acarrear la proliferación de vectores y microorganismos patógenos. Los problemas ambientales que existen se deben, sobre todo, a que las personas y las comunidades no poseen un conocimiento "objetivo" y "real" de cómo es y de cómo funciona el mundo real. Por ello, la educación en general, y la Educación Ambiental, en particular, deben centrarse en transmitir aquellos conocimientos científicos que permitan construir una imagen real de su entorno para que la gente lo perciba y entienda tal y como realmente es y, por lo tanto, les permita actuar también en consecuencia. La insistencia en convertir la Educación Ambiental en una educación esencialmente "científica" expresa muchas veces este tipo de proyectos de construcción. 


\section{Bibliografía}

Arboleda, I. F. M., \& Páramo, P. (2014). La investigación en educación ambiental en América Latina: un análisis bibliométrico. Revista Colombiana de educación, (66), 55-72.

Balcázar, F. E. (2003). Investigación acción participativa (iap): Aspectos conceptuales y dificultades de implementación. Fundamentos en humanidades, 4(7-8), 59-77.

Boff L. (2004). Saber cuidar: ética de lo humano, complejidad de la vida para la vida, 11. Ed. Petrópolis-RJ. Editora Vozes. 88 pp.

Castillo, R. M. (2010). La importancia de la educación ambiental ante la problemática actual. Revista Electrónica Educare, 14(1), 97-111.

Duque Pérez, F. A. (2011). La educación ambiental para la conservación del agua en el Consejo Popular de Jibacoa (Doctoral dissertation, Universidad Central "Marta Abreu" de Las Villas).

Escudero, J. M., \& Martínez, B. (2011). Educación inclusiva y cambio escolar. Revista iberoamericana de educación, 55, 85-105.

Frabboni, S., Gazzadi, G. C., \& Spessot, A. (2007). TEM study of annealed Pt nanostructures grown by electron beam-induced deposition. Physica E: Low-dimensional Systems and Nanostructures, 37(1-2), 265-269.

Gaudiano, E. G. (2000). Complejidad en Educación Ambiental. Tópicos en educación ambiental, 2(4), 21-32.

Guerrero, E. M., Wagner, L. S., Rodríguez, C. I., \& Sosa, B. S. (2015). Acciones colectivas y recursos naturales en conflicto. Historia ambiental de luchas urbanas por agua y sierras en Tandil, Argentina. Revista Luna Azul, (41).

González-Gaudiano, E. (2007). Schooling and environment in Latin America in the third millennium. Environmental Education Research, 13(2), 155-169.

Maass, J. M., Balvanera, P., Castillo, A., Daily, G. C., Mooney, H. A., Ehrlich, P. ... \& MartínezYrizar, A. (2005). Ecosystem services of tropical dry forests: insights from long-term ecological and social research on the Pacific Coast of Mexico. Ecology and society, 10(1).

Molina, J. F., \& Tarí, J. J. (2007). Environmental management and firm performance: A case study. Journal of environmental Management, 84(4), 606-619.

Mora, S., \& Escanero-Marcén, J. F. (2013). Análisis del ambiente educacional tras la implantación del Plan de Bolonia en la Facultad de Medicina de la Universidad de Zaragoza, España: comparación con la Facultad de Medicina de la Universidad de Chile. FEM: Revista de la Fundación Educación Médica, 16(3), 167-179.

Meira Cartea, P. Á. (2013). Problemas ambientales globales y educación ambiental: Una aproximación desde las representaciones sociales del cambio climático². Revista Integra Educativa, 6(3), 2964

Novo, M. (2009). La educación ambiental, una genuina educación para el desarrollo sostenible Environmental Education, a genuine education for sustainable development. Revista de educación, 195-217.

PNUMA, Loja, M. de, \& Internacional, N. y C. (2007). Perspectivas del Medio Ambiente Urbano: GEO Loja. Loja. Retrieved from http://www.naturalezaycultura.org/docs/Geo Loja.pdf

Pintado Astudillo, M. (2011). Elaboración de una guía para el manejo de los desechos sólidos de Rioverde, año 2011.

Torres Ibarra, L. T., \& Villalta Zelaya, Y. V. (2009). Eficacia de la protección de los recursos hídricos, como un mecanismo para garantizar el derecho humano al agua, en el Departamento de San Salvador (Doctoral dissertation, Universidad de El Salvador).

Zubieta, E. M., \& Delfino, G. (2010). Satisfacción con la vida, bienestar psicológico y bienestar social en estudiantes universitarios de Buenos Aires. Anuario de investigaciones, 17, 277-283. 